\title{
Isolated torsion of the fallopian tube in a postmenopausal woman; a rare entity
}

\author{
Murat Bozkurt, ${ }^{1}$ Duygu Kara ${ }^{2}$
}

\begin{abstract}
Keywords: fallopian tube diseases, abdominal pain, adnexal diseases, torsion abnormality, postmenopause
\end{abstract}

\begin{abstract}
Isolated torsion of the fallopian tube is an uncommon cause of acute lower abdominal pain. The lack of pathognomonic symptoms, clinical findings on physical examination, and specific imaging or laboratory characteristics makes this entity difficult to diagnose preoperatively, which can cause surgical intervention to be postponed. We report a case of isolated torsion of the right fallopian tube in a 58-year-old woman with a history of sexually transmitted infection. In most reported cases, there is no vascular flow on Doppler imaging modalities. We also detected high impedance of vascular flow around the cyst wall. Imaging modalities could not provide a definitive diagnosis. Tubal torsion was diagnosed upon laparotomy.
\end{abstract}

${ }^{1}$ Department of Obstetrics and Gynecology, Universal Hospitals Group, Malatya, Turkey ${ }^{2}$ Department of Radiology, Mus State Hospital, Mus, Turkey

\section{INTRODUCTION}

Isolated torsion of the fallopian tube is an uncommon cause of acute lower abdominal pain. The incidence is estimated to be 1 in 500,000 women. $^{1}$
This condition is infrequently observed before menarche or during menopause, which is mostly likely because most risk factors for tubal torsion occur mainly in reproductive age women. Most associated symptoms, such as abdominal pain, nausea, fever, bowel and bladder complaints, are non-specific. Immediate diagnosis is often difficult. The lack of pathognomonic symptoms, clinical findings on physical examination, and specific imaging or laboratory characteristics makes this entity difficult to diagnose preoperatively, which can cause surgical intervention to be postponed.

\section{CASE REPORT}

We report a case of a 58-year-old woman (gravida 4, para 3) with a history of sexually transmitted infection. Six hours before admission, she experienced severe pelvic pain, nausea and vomiting without vaginal bleeding. Her blood pressure was

\footnotetext{
Please cite this paper as: Bozkurt M, Kara D. Isolated torsion of the fallopian tube in a postmenopausal woman; a rare entity. Proc Obstet Gynecol. 2013;3(2):Article 6 [ 7 p.]. Available from: http://ir.uiowa.edu/pogl. Free full text article.
}

Corresponding author: Murat Bozkurt, Universal Malatya Hastanesi, Turgut Ozal Bulvari Ankara Asfalti 6.km No:216 44000 Malayta/Turkey. Telephone 904222382828/1546. Fax: 904222382600. jindrmb@yahoo.com.

Financial Disclosure: The authors report no conflict of interest.

Received: 2 August 2012; received in revised form: 22 October 2012; accepted 15 November 2012; final revisions accepted 28 February 2013; POG in Press, 13 March 2013; published online, 3 July 2013.

This is an Open Access article distributed under the terms of the Creative Commons Attribution 3.0 Unported License (http://creativecommons.org/licenses/by/3.0), which permits unrestricted use, distribution, and reproduction in any medium, provided the original work is properly cited. 
$110 / 70 \mathrm{~mm} \mathrm{Hg}$ and her temperature was $36.8^{\circ} \mathrm{C}$. Abdominal palpation revealed tenderness of the right iliac fossa. Vaginal examination revealed a white discharge and several nabothian follicles on the cervix. The uterus was slightly bulky and retroverted, and its movement was restricted by a very tender, long, oval, firm mass in the right fornix in close proximity to the uterus. This mass was easily palpable and slightly mobile in the right adnexal region.

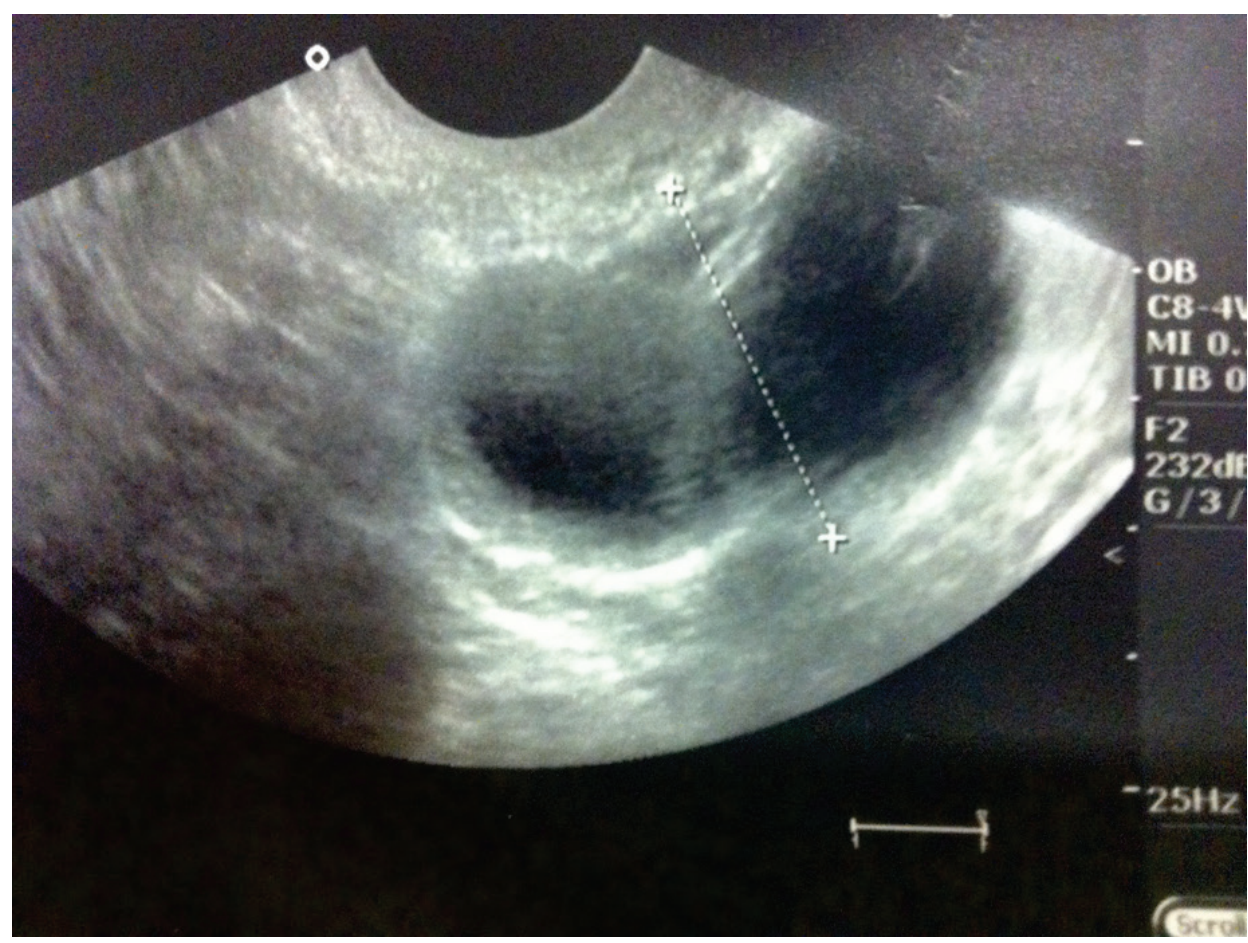

Figure 1: Ultrasound revealed a well circumscribed right adnexal cystic mass measuring $80 \times 76 \times 48 \mathrm{~mm}$ with thick septations and lobulations.

Movement of the cervix was painful and there was some tenderness in the left fornix, but no mass. Ultrasound revealed a well-circumscribed right adnexal cystic mass measuring $80 \times 76 \times 48 \mathrm{~mm}$ with thick septations and lobulations (Figure 1). Vascular flow was highly impeded around the cyst as demonstrated by Doppler examination (Figure 2). The right ovary was not identified and the left ovary was normal. White blood count was 9,200/ml; C-reactive protein was negative. Erythrocyte sedimentation rate was normal. Serum markers of ovarian malignancy were obtained and found to be within normal limits. Laparotomy was performed due to suspicion of right adnexa cystic lesion. Laparotomy showed dilated, twisted necrotic right fallopian tube that was tense and darkened in appearance, measuring 12x8 cm (Figure 3). Specifically, it was twisted clockwise three times at the infundibulo-pelvic ligament site and severely dilated. Due 
to the postmenopausal state of the patient and necrotic appearance of the tube, detorsion was not attempted and rather salpingectomy was performed. Histological examination reported a hematosalpinx with neutrophilic infiltrate, edema and dilated congested vessels in the tubal wall as well as infarction. The postoperative course was uneventful and no complications were observed at follow-up examination.

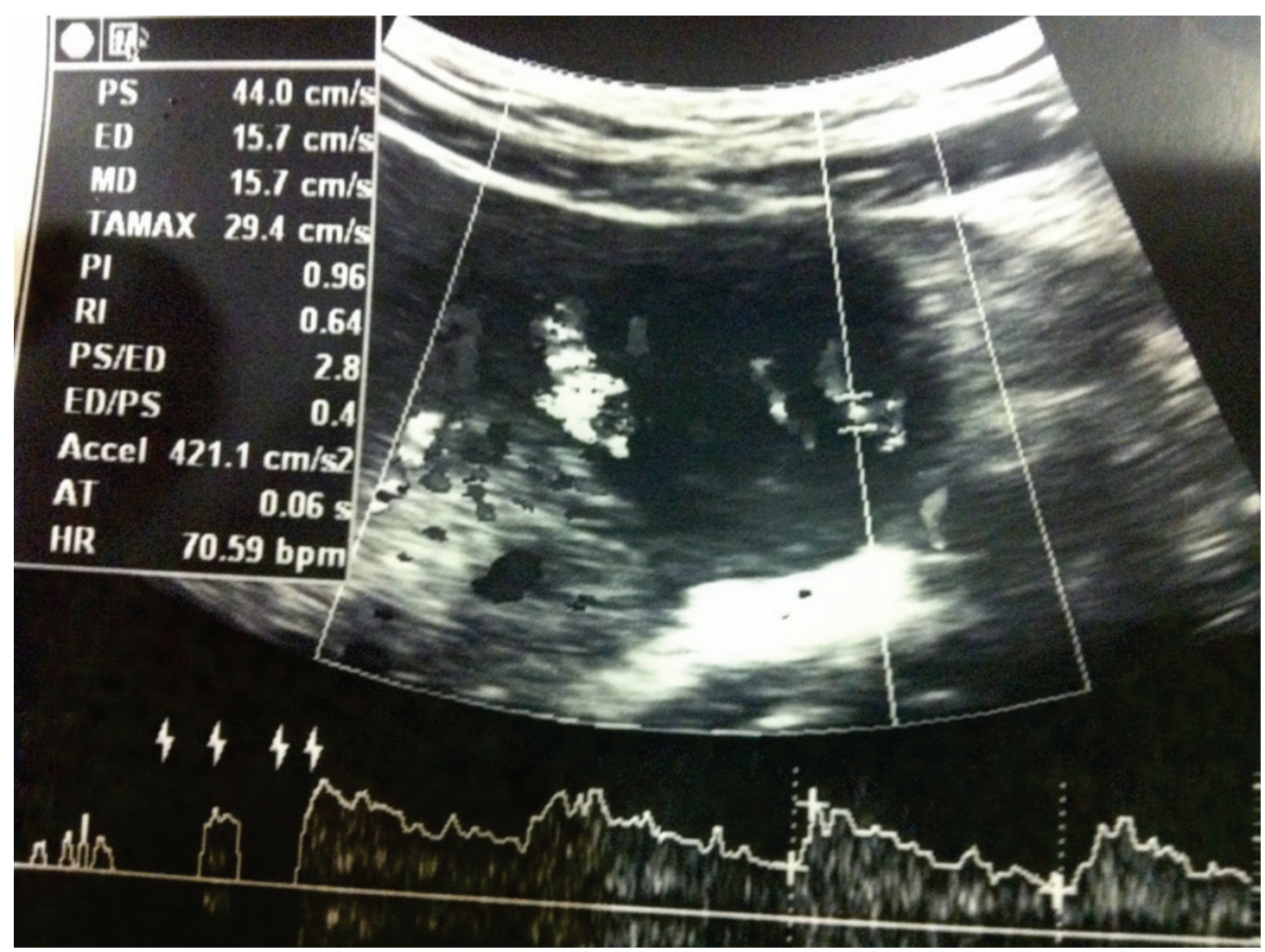

Figure 2: Doppler examination demonstrated a substantial impedance of vascular flow around the cyst.

\section{DISCUSSION}

Only sporadic cases of fallopian tube torsion have been reported. ${ }^{3}$ It rarely occurs before menarche or during menopause ${ }^{4,5}$ apparently because most risk factors for tubal torsion occur mainly in the reproductive years.

Youssef et al. described factors that could possibly influence the occurrence of fallopian tube torsion and divided them into two types: internal and external. ${ }^{6}$ (Table 1) Taken together, the existing reports indicate that the mechanism underlying tubal torsion is apparently a sequential mechanical event. ${ }^{7}$ The process begins with the mechanical blockage of the adnexal veins and lymphatic vessels by ovarian tumor, pregnancy, hydrosalpinx and pelvic adhesions after tubal infection, or pelvic operation. This obstruction causes pelvic congestion and local edema, with subsequent enlargement of the 
adnexa, which in turn induces partial or complete torsion. (Table 2) Furthermore, the mechanical blockage of the distal part of the fallopian tubes, together with the normal secretions of the fallopian tube glands, can cause hydrosalpinx, a risk factor by itself for torsion.

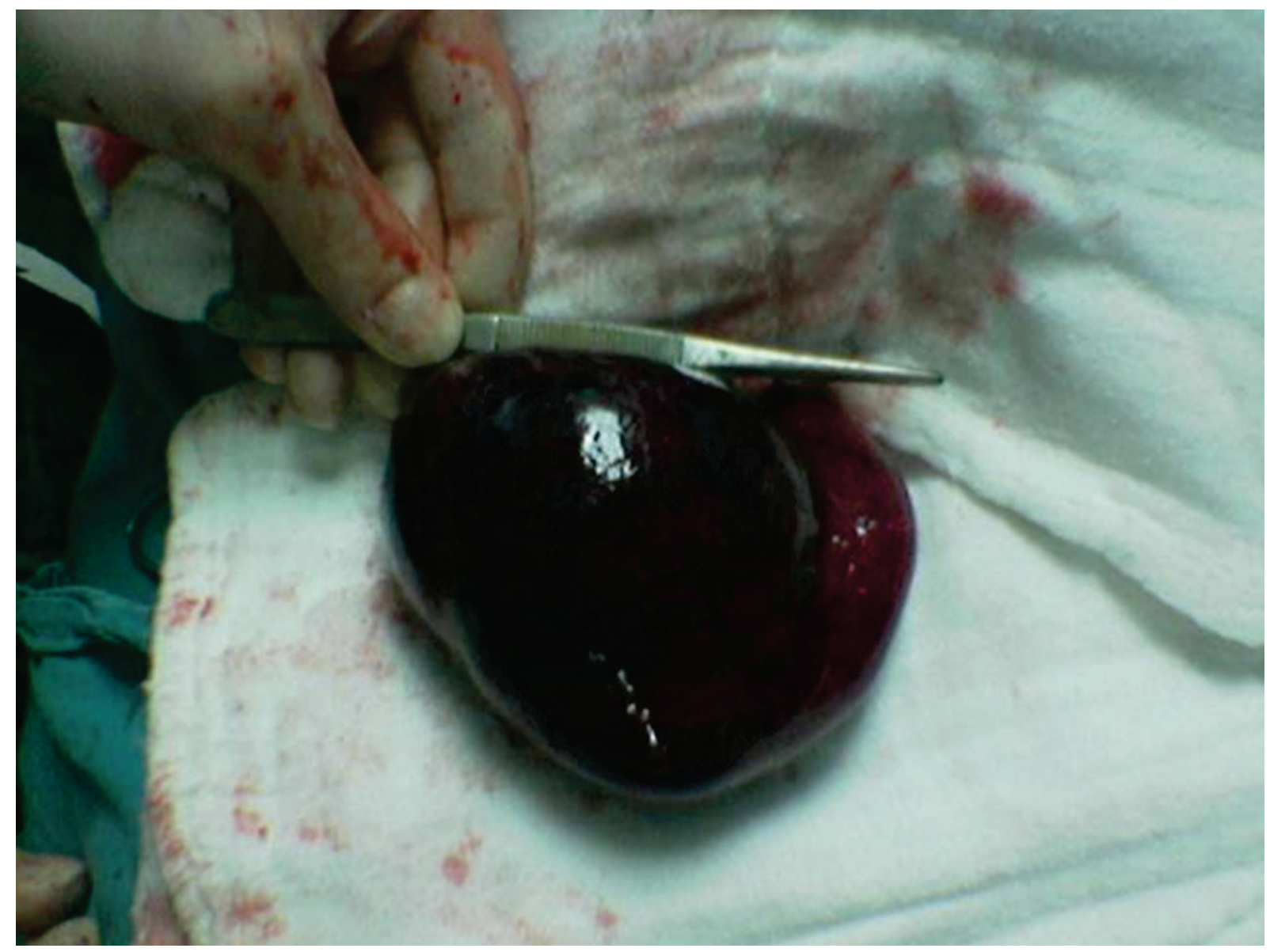

Figure 3: Dilated, twisted necrotic right fallopian tube was tense and darkened in appearance, measuring $12 \times 8 \mathrm{~cm}$.

Table 1. Intrinsic and Extrinsic Causes of Fallopian Tube Torsion

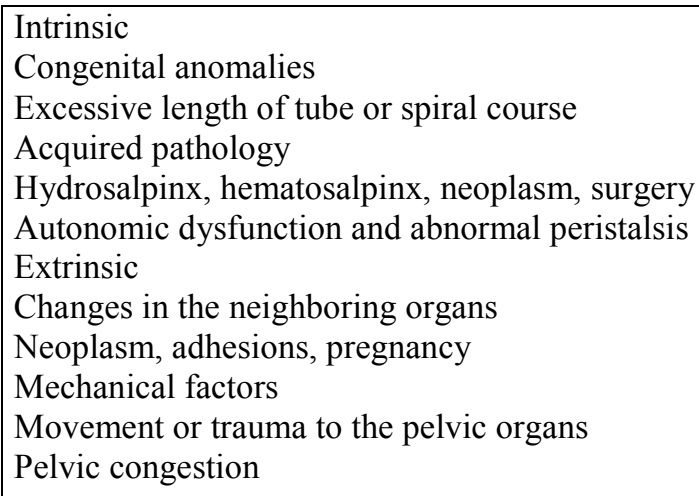

\section{Table 2. Possible Mechanisms for Tubal Torsion.}

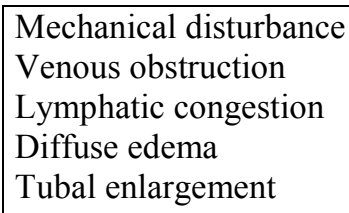

The most common symptom of tubal torsion is pain located in the lower abdominal region or pelvis that may 
radiate to the flank or thigh. Sudden onset of cramping pain or intermittent pain is possible. In the present case, there was previous history of pelvic infection, which may have contributed to the etiology. Other factors associated with tubal torsion include slightly elevated temperature, white blood cell count, and erythrocyte sedimentation rate, though these factors may also be normal. In the case presented herein, these parameters were normal. The differential diagnosis of fallopian tube torsion includes acute appendicitis, ectopic pregnancy, pelvic inflammatory disease, twisted ovarian cyst and degenerative leiomyoma., ${ }^{8,9}$ The sensitive adnexal mass and cervical motion tenderness in this case were originally thought to be due to tuboovarian abscess or pelvic inflammatory disease.

Pre-existing risk factors for isolated tubal torsion include tubal pathologies such as pelvic inflammatory disease, paratubal cyst, hydrosalpinx, congenital anomalies, ectopic pregnancies, endometriosis and tubal ligation devices, which are most common in reproductive age women. In the post-menopausal period, the most common risk factors are neoplasia or extrinsic lesions such as adhesions. $^{10}$ Tubal torsion is more frequently observed on the right side, possibly because of partial immobilization of the left fallopian tube by its proximity to the sigmoid mesentery or because right lower quadrant pain is more often surgically explored secondary to the concern for appendicitis. $^{11}$ In this case report, isolated tubal torsion occurs in the right adnexa, consistent with other reports in the literature.
Imaging findings are generally noninformative in the preoperative diagnosis of isolated torsion of the fallopian tubes. Reported sonographic findings include a normal-appearing uterus, fluid-free ovaries with normal flow, a dilated tube with thickened, echogenic walls; and internal debris or a convoluted echogenic mass thought to represent a thickened, twisted tube. Our finding of high impedance of flow in a tubular structure, especially in a patient without a history of tubal ligation, does not necessarily indicate fallopian tube torsion. ${ }^{12}$ The added value of color Doppler sonography in the diagnosis of adnexal torsion has not been fully established. In several studies with a small numbers of patients, investigators have concluded that the diagnosis or exclusion of adnexal torsion cannot be reliably based on the absence or presence of flow on color Doppler sonography. Those authors remarked that normal blood flow commonly is seen in twisted adnexa. The identification of a whirlpool sign on Doppler sonography has been suggested to be pathognomonic of adnexal torsion; however, this sign is not commonly seen. ${ }^{13,14}$ The whirlpool sign was determined as a clockwise or counterclockwise wrapping of the hypoechoic vessels around the central axis. In our case, sonographic evaluation revealed a wellcircumscribed right adnexal cystic mass, with thick septations and lobulations. On Doppler examination high impedance of vascular flow was detected around the cyst, but the whirlpool sign was not observed. The finding of high impedance of flow tubular structure, especially in a patient with no history of tubal ligation, cannot be used as an indicator of fallopian tube torsion. In most cases, there is no 
vascular flow on Doppler imaging modalities. Other radiologic modalities such as computed tomography or magnetic resonance imaging have also been reported to be valuable for the diagnosis of tubal torsion. ${ }^{15}$ Diagnosis of an isolated tubal torsion based on imaging results is far more difficult because of the lack of specific findings. CT is insufficient for accurate detection and prediction of the nature of an underlying pathologic process causing torsion. MRI findings yield the same information as CT and US. In this presented case, due to the patient's state of emergency CT and MRI evaluation were not performed.

Treatment options of the isolated tubal torsion include detorsion or simple salpingectomy, depending on the timing of surgical intervention and presence of complications. The risk of thromboembolic events is extremely low when the adnexal detorsion can be performed as early as possible. However, it should be performed as early as possible to avoid irreversible damage to the tissue. The operative approach could be conventional exploratory laparotomy or laparoscopic surgery. Currently, laparoscopic adnexal detorsion, rather than adnexectomy, is the procedure of choice. Because most of the patients are in their reproductive years, efforts should be made to preserve fertility if the ischemic damage appears to be reversible and no malignancy is suspected. A complete resection is performed when the tissue is gangrenous, there is a tubal or ovarian neoplasm, or the woman has no desire for future conception. When there is no apparent ischemic damage, most of the twisted adnexa regain function. In our case salpingectomy was performed because the patient was in the post- menopausal period, though no malignancy was suspected.

\section{CONCLUSION}

In this case presentation, we emphasize that isolated tubal torsion should be considered in the differential diagnosis of acute lower abdominal pain and adnexal masses in the postmenopausal patients. In most reported cases, there is no vascular flow on Doppler imaging modalities. We also detected high impedance of vascular flow around the cyst wall. İmaging modalities could not provide a definitive diagnosis.

\section{References}

1. Hansen $\mathrm{OH}$. Isolated torsion of the Fallopian tube. Acta Obstet Gynecol Scand. 1970;49(1):3-6. PubMed PMID: $\quad 5519474$. http://dx.doi.org/10.3109/00016347009 157506

2. Ding DC, Hsu S, Kao SP. Isolated torsion of the hydrosalpinx in a postmenopausal woman. JSLS. 2007 Apr-Jun;11(2):252-4. PubMed PMID: 17761091; PubMed Central PMCID: PMC3015729.

3. Shukla $R$. Isolated torsion of the hydrosalpinx: a rare presentation. $\mathrm{Br} \mathrm{J}$ Radiol. $2004 \quad$ Sep;77(921):784-6. PubMed PMID: 15447969. http://dx.doi.org/10.1259/bjr/36288287

4. Krissi H, Shalev J, Bar-Hava I, Langer R, Herman A, Kaplan B. Fallopian tube torsion: laparoscopic evaluation and treatment of a rare gynecological entity. J Am Board Fam Pract. 2001 Jul-Aug;14(4):274-7. Review. PubMed PMID: 11458970. 
5. Feng L, Liu Y, Liu H, Chen X. Chronic isolated fallopian tube torsion associated with huge hydrosalpinx and hemosalpinx in a postmenopausal woman: a case report and brief review. Eur J Obstet Gynecol Reprod Biol. 2012 Oct;164(2):235-6. doi: 10.1016/j.ejogrb.2012.06.011. Epub 2012 Jun 30. PubMed PMID: 22749784.

6. Youssef AF, Fayad MM, Shafeek MA. Torsion of the fallopian tube. A clinicopathological study. Acta Obstet Gynecol Scand. 1962;41:292-309. PubMed PMID: 14002859. http://dx.doi.org/10.3109/00016346209 158105

7. Bernardus RE, Van der Slikke JW, Roex AJ, Dijkhuizen GH, Stolk JG. Torsion of the fallopian tube: some considerations on its etiology. Obstet Gynecol. 1984 Nov;64(5):675-8. PubMed PMID: 6238251.

8. Wong SW, Suen SH, Lao T, Chung $\mathrm{KH}$. Isolated fallopian tube torsion: a series of six cases. Acta Obstet Gynecol Scand. 2010 Oct;89(10):1354-6. doi: 10.3109/00016349.2010.503870. PubMed PMID: 20726829.

9. Ferrera PC, Kass LE, Verdile VP. Torsion of the fallopian tube. Am J Emerg Med. 1995 May;13(3):312-4. PubMed PMID: 7755826. http://dx.doi.org/10.1016/07356757(95)90208-2
10. Comerci G, Colombo FM, Stefanetti M, Grazia G. Isolated fallopian tube torsion: a rare but important event for women of reproductive age. Fertil Steril. 2008 Oct;90(4):1198.e23-5. Epub 2007 Oct 22. PubMed PMID: 17953952.

http://dx.doi.org/10.1016/j.fertnstert.20 07.08.057

11. Bondioni MP, McHugh K, Grazioli L. Isolated fallopian tube torsion in an adolescent: CT features. Pediatr Radiol. $2002 \quad$ Aug;32(8):612-3. PubMed PMID: 12269256. http://dx.doi.org/10.1007/s00247-0020721-8

12. Propeck PA, Scanlan KA. Isolated fallopian tube torsion. AJR Am J Roentgenol. 1998 Apr;170(4):1112-3. PubMed PMID: 9530080.

13. Albayram F, Hamper UM. Ovarian and adnexal torsion: spectrum of sonographic findings with pathologic correlation. J Ultrasound Med. 2001 Oct;20(10):1083-9. PubMed PMID: 11587015.

14. Wachsberg RH. Accuracy of using Doppler sonography to diagnose ovarian torsion. Fertil Steril. 2001 May;75(5):1041-2. PubMed PMID: 11368055.

http://dx.doi.org/10.1016/S00150282(01)01763-0

15. Terada $Y$, Murakami T, Nakamura $S$, Sato $\mathrm{Y}$, Niikura H, Ito K, Yaegashi N, Okamura K. Isolated torsion of the distal part of the fallopian tube in a premenarcheal 12 year old girl: a case report. Tohoku J Exp Med. 2004 Mar;202(3):239-43. PubMed PMID: 15065650.

http://dx.doi.org/10.1620/tjem.202.239 